EPJ Web of Conferences 62, 07002 (2013)

DOI: $10.1051 /$ epjconf/20136207002

(C) Owned by the authors, published by EDP Sciences, 2013

\title{
Ternary fission induced by polarized neutrons
}

\author{
Friedrich Gönnenwein \\ Physikalisches Institut, Universität Tübingen, 72076 Tübingen, Germany
}

\begin{abstract}
Ternary fission of (e,e) U- and $\mathrm{Pu}$ - isotopes induced by cold polarized neutrons discloses some new facets of the process. In the so-called ROT effect shifts in the angular distributions of ternary particles relative to the fission fragments show up. In the so-called TRI effect an asymmetry in the emission of ternary particles relative to a plane formed by the fragment momentum and the spin of the neutron appear. The two effects are shown to be linked to the components of angular momentum perpendicular and parallel to the fission axis at the saddle point of fission. Based on theoretical models the spectroscopic properties of the collective transitional states at the saddle point are inferred from experiment.
\end{abstract}

\section{Experiments}

In analogy to the search for violation of time reversal invariance in the decay of free polarized neutrons it was suggested that that to that purpose also ternary fission could be an appropriate reaction. The T-odd variable to be scrutinized is the triple correlation

$$
\mathrm{B}=\sigma_{\mathrm{n}} \cdot\left[\mathbf{p}_{\mathrm{LF}} \mathrm{x} \mathbf{p}_{\mathrm{TP}}\right]=\mathbf{p}_{\mathrm{TP}} \cdot\left[\sigma_{\mathrm{n}} \mathrm{x} \mathbf{p}_{\mathrm{LF}}\right]
$$

with $\boldsymbol{\sigma}_{\mathrm{n}}$ the neutron spin, $\mathbf{p}_{\mathrm{LF}}$ and $\mathbf{p}_{\mathrm{TP}}$ the momenta of the light fragment LF and the ternary particle TP. All vectors are understood to be unit vectors. The correlation B changes sign upon time reversal. In case a correlation $\mathrm{B} \neq 0$ is observed this may indicate a violation of time reversal invariance. However, unlike neutron decay the condition $\mathrm{B} \neq 0$ is necessary but not sufficient.

The correlator becomes maximal with $\mathrm{B}= \pm 1$ when all three vectors of $\mathrm{B}$ are orthogonal to each other. This is achieved in a layout of the experiments where the neutron beam traversing the target is polarized longitudinally either in or against neutron direction of flight, and the detectors for fission fragments and ternary particles are centered in a plane perpendicular to the beam at right angles relative to each other. Fission fragments were intercepted by two position-sensitive multi-wire-proportionalcounters facing each other on opposite sides of the target mounted in a horizontal plane, while ternary particles were measured by two arrays of up to $19 \mathrm{PIN}$ diodes facing each other in a vertical plane. The fissile targets studied were ${ }^{233} \mathrm{U},{ }^{235} \mathrm{U},{ }^{239} \mathrm{Pu}$ and ${ }^{241} \mathrm{Pu}$.

The cold neutron beam (flux $\Phi \approx 3 \cdot 10^{8} / \mathrm{cm}^{2} \cdot \mathrm{s}$, wavelength $\langle\lambda\rangle=0.45 \mathrm{~nm}$ ) was polarized longitudinally (polarization $>95 \%$ ) and was flipped every second parallel or anti-parallel to the neutron direction of flight. For fixed neutron spin helicity $\boldsymbol{\sigma}_{+}$or $\boldsymbol{\sigma}_{-}$coincidences between fragments and ternary particles were taken. In the evaluation the basic quantity is the asymmetry $\mathrm{A}$ in the count rates $\mathrm{N} \uparrow$ and

This is an Open Access article distributed under the terms of the Creative Commons Attribution License 2.0, which permits unrestricted use, distribution, and reproduction in any medium, provided the original work is properly cited. 


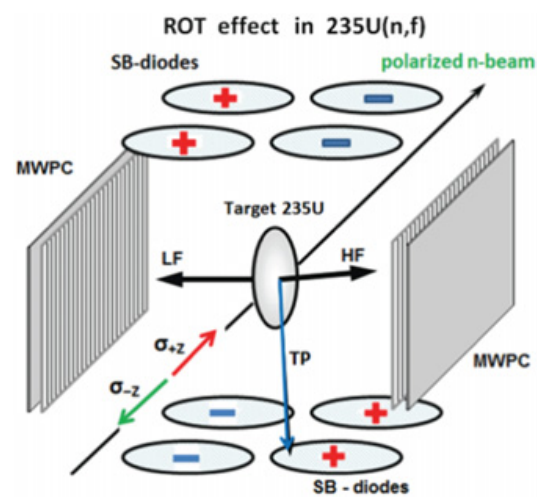

Figure 1. ROT effect in ${ }^{235} \mathrm{U}(\mathrm{n}, \mathrm{f})$.

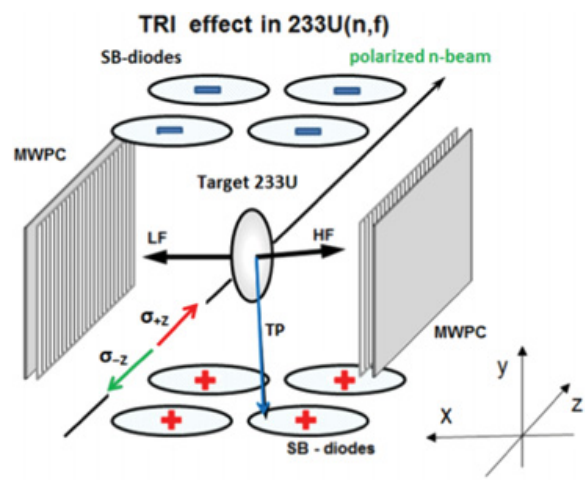

Figure 3. TRI effect in ${ }^{233} \mathrm{U}(\mathrm{n}, \mathrm{f})$.

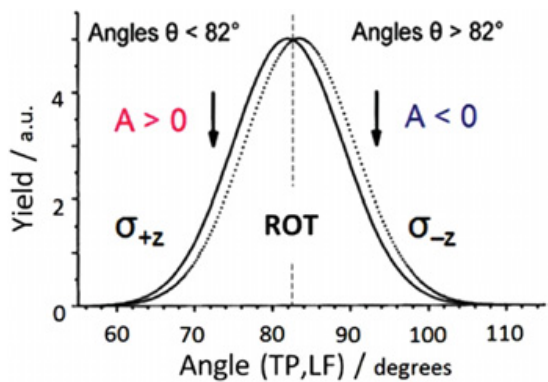

Figure 2. Phenomenology of ROT effect.

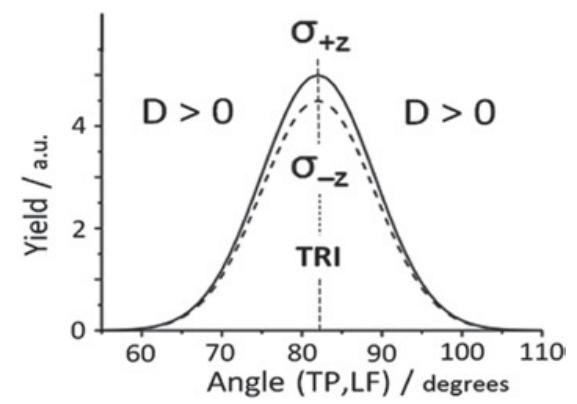

Figure 4. Phenomenology of TRI effect.

$\mathrm{N} \downarrow$ for the two spin positions $\boldsymbol{\sigma}_{+}$and $\boldsymbol{\sigma}_{-}$, respectively, defined as

$$
\mathrm{A}=(\mathrm{N} \uparrow-\mathrm{N} \downarrow) / / \mathrm{N} \uparrow+\mathrm{N} \downarrow) .
$$

For the two reactions ${ }^{235} \mathrm{U}(\mathrm{n}, \mathrm{f})$ and ${ }^{233} \mathrm{U}(\mathrm{n}, \mathrm{f})$ surprisingly two distinct patterns of the signs for the asymmetry A were observed as shown in the Figures 1 and 3. They were named ROT- and TRI-effect, respectively.

In the ROT effect for the reaction ${ }^{235} \mathrm{U}(\mathrm{n}, \mathrm{f})$ the signs of the asymmetry A indicated in Figure 1 depend on the angle $\theta$ between TP and LF. The signs switch for $\theta<82^{\circ}$ and $\theta>82^{\circ}$. This is in disagreement with expectation for the correlation B of Eq. (1). In a phenomenological approach the sign pattern is understood as a shift of the Gaussian-shaped angular distribution $\mathrm{W}(\theta)$ of TPs, which is centered at $\theta \approx 82^{\circ}$, to smaller or larger angles depending on neutron spin. The asymmetry from Eq. (2) indeed has opposite signs for $\theta$ angles in the two cases. This is visualized in Figure 10.

In the reaction ${ }^{233} \mathrm{U}(\mathrm{n}, \mathrm{f})$ the signs of the asymmetry A do not depend on the angle $\theta$ between TP and LF but change for the TP flying either parallel or antiparallel to $\left[\boldsymbol{\sigma}_{\mathrm{n}} \times \mathbf{p}_{\mathrm{LF}}\right]$ as conjectured by the correlator B. This feature is on display in Figure 3. Again in a phenomenological approach this is described as a dependence of the TP yield on the spin orientation in Figure 4. 


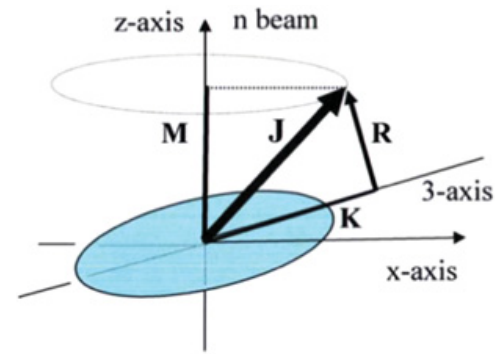

Figure 5. J, K, R scheme.
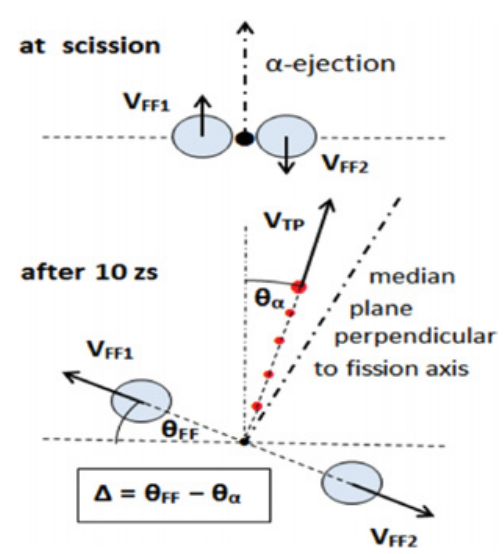

Figure 6. Model of ROT effect.

\section{Model of the ROT effect}

For thermal neutron fission of the fissile nuclei ${ }^{233,235} \mathrm{U}$ and ${ }^{239,241} \mathrm{Pu}$ presently studied all the available states at the saddle point of fission are collective states. In a semi-classical picture the components $\mathbf{K}$ and $\mathbf{R}$ of the total angular momentum $\mathbf{J}$ parallel and perpendicular to $\mathbf{J}$ are hence collective rotations. It is then tempting to trace the two types of asymmetry A to the two types of collective rotations. The breakdown of $\mathbf{J}$ into $\mathbf{K}$ and $\mathbf{R}$ is visualized in Figure 5.

In particular the ROT effect is understood in terms of ternary particles being ejected at scission from a nucleus rotating with angular momentum $\mathbf{R}$. As illustrated in Figure 6, symmetric fission with the ternary particle emitted perpendicular to the fission axis is considered. After scission the rotation strictly speaking ceases. But since at start the fragments are expelled from a rotating compound, the fission axis connecting the two fragments continues to turn around after scission. By inertia the fragments fly in opposite directions on straight parallel paths. However, Coulomb repulsion between fragments is pushing them further apart. As to ternary fission, for the scission configuration in Figure 6 the Coulomb forces try to keep the ternary particle in the median plane perpendicular to the fission axis. The ternary particle is thus entrained in the turning of the fission axis. But since the Coulomb forces become weaker as time goes on and the distances between particles increase, the ternary particle is no longer sticking to the median plane and the angle $\theta_{\alpha}$ of the ternary particle falls behind the turning angle $\theta_{\mathrm{FF}}$ of the fission axis. The lag angle is called $\Delta$ :

$$
\Delta=\theta_{\mathrm{FF}}-\theta_{\alpha} .
$$

The angle $\Delta$ is precisely the shift of angular distributions of ternaries in fission induced by polarized neutrons compared to un-polarized neutrons. As argued in connection with Figure 10, the angular shift $\Delta$ is observed in experiment as an asymmetry called ROT effect.

The phenomenological model of Figure 6 has been corroborated by trajectory calculations for fragments and ternary particles emanating from a rotating scission configuration. First, starting conditions reproducing best energy and angular distributions of ternary particles for a non-rotating nucleus were determined and then generalized to take into account the rotation of the fissioning system at start. Results from these calculations are given in the Figures 7 and 8.

In Figure 7 the angular velocity of turning $\mathrm{d} \theta_{\mathrm{FF}} / \mathrm{dt}$ of the fission axis (more precisely: the axis from the center of mass to the light fragment) as a function of time is plotted for an assumed average angular momentum $\langle\mathrm{Jz}\rangle=1 \hbar$. It is seen that the angular velocity approaches zero in a few zs. This is understood 


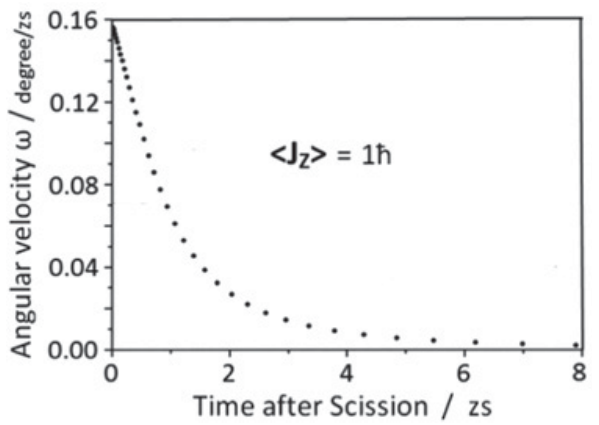

Figure 7. Angular velocity of fission axis.

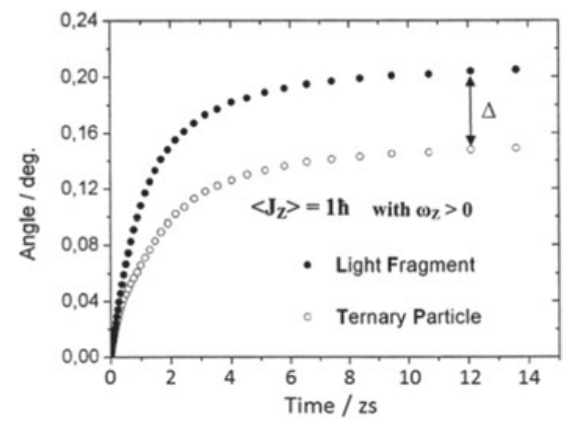

Figure 8. Turning angle of LF and Ternary $\alpha$-Particle.

by noting that within a few zs all particles have moved far apart and at constant angular momentum the increase of the moment of inertia will hence slow down the angular velocity.

In Figure 8 the turning angles $\theta_{\mathrm{FF}}(\mathrm{t})$ and $\theta_{\mathrm{TP}}(\mathrm{t})$ of the light fission fragment and the ternary particle are displayed as a function of time after scission. Both angles become stationary in a few zs. As suggested phenomenologically in Figure 6 the lag angle $\Delta$ from Eq. (3) between $\theta_{\mathrm{FF}}$ and $\theta_{\mathrm{TP}}$ is nonzero albeit very small: $\theta \approx 0.04^{\circ}$.

More in detail the trajectory calculations allow to assess the quantum numbers $\mathrm{J}, \mathrm{K}$ and $\mathrm{R}$ of the transition states at the saddle point of a fissioning nucleus. This requires the knowledge of the angular velocity $\omega_{z}(\mathbf{J}, \mathbf{K})$ at scission as starting conditions for the trajectory calculations. The angular velocity has been derived quantum-mechanically [1]. First, the polarization $\mathrm{P}(\mathrm{J}, \mathrm{K})$ of the nucleus after capture of a polarized neutron $\mathrm{P}(\mathrm{J}, \mathrm{K})=\langle\mathrm{J}(\mathrm{K})\rangle / \mathrm{J}$ is found to be

$$
\begin{array}{ll}
P(J, K)=+\frac{P_{n}}{2 J} \cdot \frac{J(J+1)-K^{2}}{J} & \text { for } J=J_{+}=I-1 / 2 \\
P(J, K)=+\frac{P_{n}}{2 J} \cdot \frac{J(J+1)-K^{2}}{J+1} & \text { for } J=J_{-}=I-1 / 2
\end{array}
$$

with $\mathrm{p}_{\mathrm{n}}$ the neutron polarization, I the target and $\mathrm{J}$ the compound spin. The angular velocity then is

$$
\omega_{\mathrm{z}}(J, K)=\frac{\left\langle J_{z}(J, K)\right\rangle}{\mathfrak{\Im}_{\perp}}=\frac{J \cdot P(J, K)}{\mathfrak{\Im}_{\perp}}
$$

where $\mathfrak{I}_{\perp}$ is the moment of inertia. Calculational results for the shift difference $2 \Delta$ between shifts for the two neutron spin orientations are shown in Figure 9 for the reaction ${ }^{233} U\left(n_{t h}, f\right)$. Note that $2 \Delta$ is the shift of angular $\alpha$-distributions being sensed by the spin flip technique. For ${ }^{233} \mathrm{U}$ the target has $\mathrm{I}=7 / 2 \hbar$ and the compound spin is $J=4 \hbar$ or $3 \hbar$. For any angular velocity $\omega_{z}(J, K)$ the shift difference $2 \Delta$ may be found as a function of the final kinetic energy of the ternary $\alpha$-particle $\mathrm{E}_{\alpha}$, as plotted in Figure 9 . It has to be pointed to the change of sign for $J_{+}=I+1 / 2$ and $J_{-}=I-1 / 2$. Further, the size of the angular shift is largest for $K=0$ and, since $R(R+1)=J(J+1)-K^{2}$, largest for maximal $R=J$, i.e. for the maximum orbital rotation $\mathrm{R}$ perpendicular to the fission axis. This is in line with the expectation suggested by the model for the ROT effect in Figure 6.

In experiment the two spin states $\mathrm{J}_{+}$and $\mathrm{J}_{-}$contributing to fission can not be disentangled and therefore an effective shift $2 \Delta$ weighted by the respective fission cross sections is observed. The asymmetry A from Eq. (2) is according to figure 10 depending on the angle between LF and TP. The asymmetry is compared in Figure 9 for the reaction ${ }^{233} \mathrm{U}(\mathrm{n}, \mathrm{f})$ as observed in experiment (open circles) and as fitted by the model outlined (squares) assuming that there is only one quantum state per 


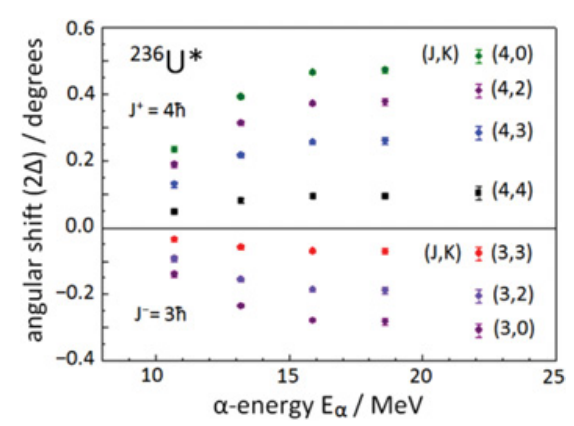

Figure 9. Shift $2 \Delta$ versus quantum numbers $(\mathrm{J}, \mathrm{K})$.

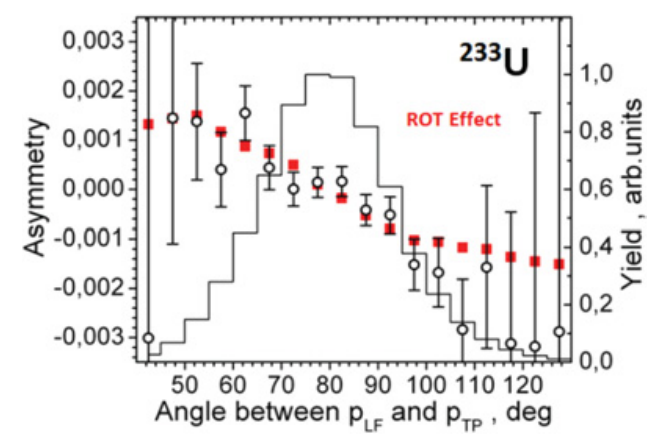

Figure 10. Asymmetry A for ${ }^{233} U(n, f)$ in experiment and from model versus angle $\left(\mathbf{p}_{\mathrm{LF}}, \mathbf{p}_{\mathrm{TP}}\right)$.

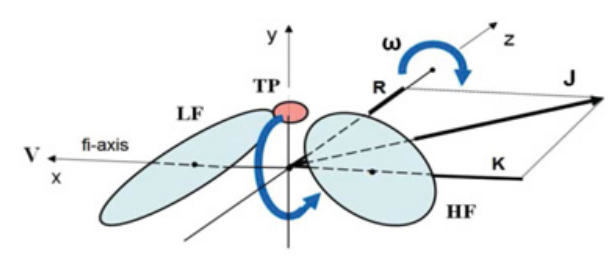

Figure 11. Model for the TRI effect.

capture spin. The model yields an excellent fit to the data for the combination $\left(\mathrm{J}_{+}, \mathrm{K}_{+}\right)=(4,0)$ and $\left(\mathrm{J}_{-}, \mathrm{K}_{-}\right)=(3,2)$ as a function of angle between $\mathbf{p}_{\mathrm{LF}}$ and $\mathbf{p}_{\mathrm{TP}}$. The role of the sate $\mathrm{K}=0$ or $\mathrm{R}=\mathrm{J}$ is emphasized, playing to all evidence a pivotal role for the ROT effect.

\section{Model for the TRI effect}

Having demonstrated that the ROT effect is to be attributed to the R-component of angular momentum $\mathrm{J}$, it is conjectured that the TRI effect may be linked to the projection $\mathrm{K}$ of $\mathrm{J}$ on the fission axis. In fission of fissile nuclei induced by slow neutrons as the one studied here, all states at the saddle point of fission are collective. But there is a difficulty. Collective states around the fission axis with component $\mathrm{K}$ (see Fig. 5) along the fission axis are only feasible for a nucleus with broken axial symmetry. A nuclear model with these properties is proposed.

Non-axial oscillations of a nucleus near the scission point were invoked long ago in the theory of fragment angular momentum [2]. The modes in question are the bending or butterfly modes sketched in Figure 11. In fact there are two degenerate linear vibration modes perpendicular to each other, one in the $(\mathrm{x}, \mathrm{y})$ plane and another in the $(\mathrm{x}, \mathrm{z})$ plane of the figure. The linear vibrations may equally well be described as two rotations around the $\mathrm{x}$-axis of the figure connecting the centers of mass of the two main fragments. The two rotations have opposite sense of rotation with angular momenta $+\mathrm{K}$ and $-\mathrm{K}$ along the fission axis. The motion is reminiscent of a "rotating banana". Note that on average the angular momentum around the fission axis is vanishing, since $+\mathrm{K}$ and $-\mathrm{K}$ have equal probabilities.

The TP is assumed to sit in the neck between the tips of the two fission prone fragments. It participates in the banana rotation. The always present orbital rotation $\mathrm{R}$ perpendicular to the fission axis imparts the system an additional $\omega$ around the z-axis in Figure 11. While due to the banana motion the TP is moving with velocity $\mathbf{v}_{\mathrm{TP}}$ up- or downwards along the y-axis of the figure, it experiences a Coriolis force $\mathbf{F} \sim\left[\mathbf{v}_{\mathrm{TP}} \mathrm{x} \boldsymbol{\omega}\right]$ ] acting along the X-axis. This force along the fission axis will either hinder 

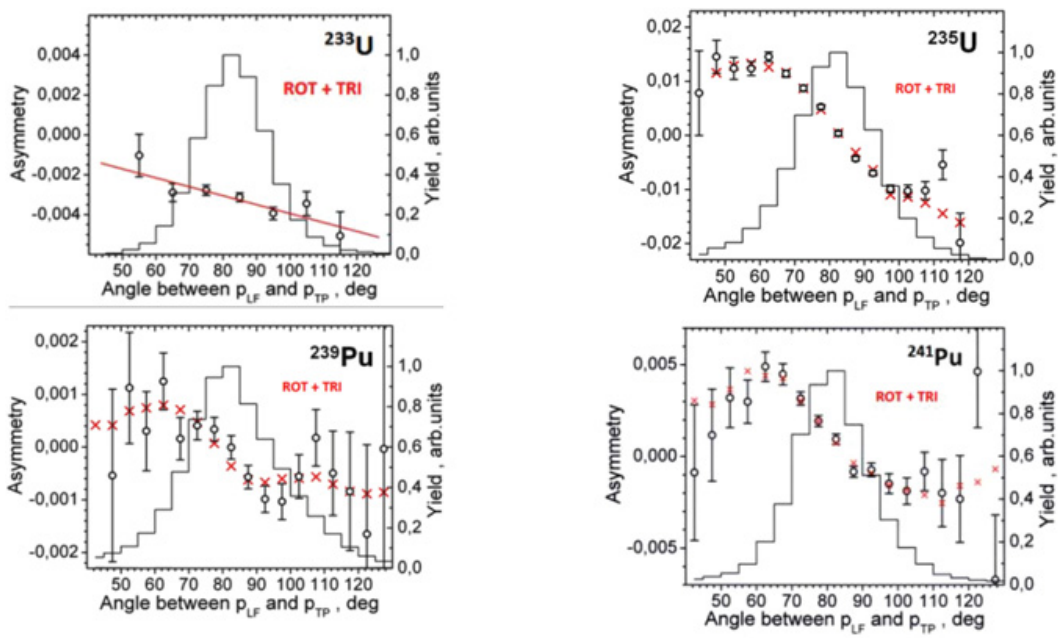

Figure 12. Asymmety $\mathrm{A}(\theta)$ : open circles experiment; crosses model; histogram angluar distribution $\mathrm{Y}(\theta)$.

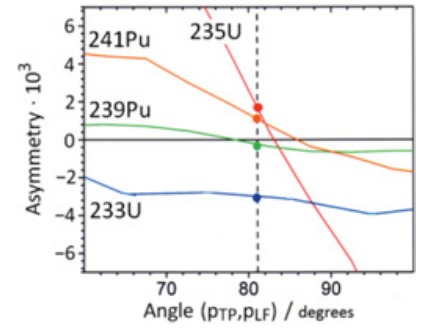

Figure 13. Asymmetry $\mathrm{A}(\theta)$. TRI effect: heavy points at $\langle\theta)\rangle=82^{\circ}$. ROT effect: sloping lines.

or favor cutting through the bond between the TP and the fragment to which it is still clinging after a first neck rupture setting free the complementary fragment. This qualitative picture explains correctly the uniform sign pattern of the asymmetry A per diode array and the change of sign between the two arrays of the experimental setup in Figure 3 characteristic for the TRI effect.

The size of the TRI effect cannot be predicted by the model. To test the consistency of the model it was therefore parameterized by replacing in the Coriolis force the velocity $\mathbf{v}_{\mathrm{TP}}$ of the ternary particle by the K-quantum number. The assumption behind is that the velocity $\mathbf{v}_{\mathrm{TP}}$ and the quantum number $\mathrm{K}$ are proportional to each other. Taking the contribution by both capture states $\mathrm{J}_{+}$and $\mathrm{J}_{-}$into account the size D of the TRI effect is with the spin separated fission cross section $\sigma\left(\mathbf{J}_{+}\right)$and $\sigma\left(\mathbf{J}_{-}\right)$

$$
D=\text { const } \cdot \cdot\left[K_{+} \omega_{+} \frac{\sigma\left(j_{+}\right)}{\sigma\left(j_{+}\right)+\sigma\left(j_{-}\right)}+K_{-} \omega_{-} \frac{\sigma\left(j_{-}\right)}{\sigma\left(j_{+}\right)+\sigma\left(j_{-}\right)} .\right.
$$

The constant pre-factor $\mathrm{D}$ was determined by a fit to the formula of the experimental $\mathrm{D}=1.7 \cdot 10^{-3}$ and the $(J, K)$ values $\left(J_{+}, K_{+}\right)=(4,0)$ and $\left(J_{-}, K_{-}\right)=(3,2)$ observed in the reaction ${ }^{233} U(n, f)$. This factor is adopted for all reactions studied. The parameterization allows to find those $(\mathrm{J}, \mathrm{K})$ combinations best fitting the experimental data for the TRI effect. The agreement between the $(\mathrm{J}, \mathrm{K})$ assignments from the 
ROT and the TRI effect is remarkable. Results are given in the following:

\begin{tabular}{l|cccc} 
Target & 233U & 235U & 239Pu & 241Pu \\
ROT effect 2 & $0.03(1)^{\circ}$ & $0.215(1)^{\circ}$ & $0.020(3)^{\circ}$ & $0.047(4)^{\circ}$ \\
TRI effect D $\cdot 10^{3}$ & $-3.90(12)$ & $1.7(2)$ & $-0.23(9)$ & $1.30(15)$ \\
$\left(\mathrm{J}_{+}, \mathrm{K}_{+}\right) ;\left(\mathrm{J}_{-}, \mathrm{K}_{-}\right)$ & $(3,2) ;(2,0)$ & $(4,0) ;(3,2)$ & $(1,1 ;(0,0)$ & $(3,0) ;(2,1)$
\end{tabular}

\section{Summary}

The asymmetries $\mathrm{A}(\theta)$ as a function of angle $\theta$ found in experiment receive in general contributions from both, the ROT and the TRI effect, i.e. respectively

$$
\mathrm{A}(\theta)=(2 \Delta) \cdot\left(\mathrm{Y}^{\prime}(\theta) / 2 \mathrm{Y}(\theta)\right)+\mathrm{D}
$$

with $\mathrm{Y}(\theta)$ the angular distribution of the TP. The ROT effect depends on the angle $\theta$ and vanishes at the average angle $\langle\theta\rangle=82^{\circ}$ where $\mathrm{Y}^{\prime}(\theta)=0$. The ROT asymmetries are described by trajectory calculations for particles in free space being ejected from a system rotating up to scission. The ROT effect is connected to the R-component of angular momentum $\mathbf{J}$. In contrast to the suggested triple correlation B in Eq. (1) no angular dependence could be disclosed for the TRI effect. It is linked to the $\mathrm{K}$-component of angular momentum $\mathbf{J}$ via the Coriolis force in the rotating fission prone system.

The results for the asymmetry $\mathrm{A}(\theta)$ are concisely suummarized in Figure 13 . The asymmetries at the angle $<\theta>=82^{\circ}$ highlighted by heavy points stand for the TRI effect shifting the A $(\theta)$ data for the pure ROT effect depending on angle $\theta$. The ROT asymmetry is indicated by the sloping lines. No simple correlation between the two asymmetry effects and macroscopic properties like mass $\mathrm{A}$ or charge $\mathrm{Z}$ or fissility $\mathrm{Z}^{2} / \mathrm{A}$ of the fissioning nucleus emerges.

\section{References}

[1] V.E. Bunakov, S.G. Kadmensky, Physics of Atomic Nuclei, 74, 1655 (2010)

[2] J.O. Rasmussen, W. Nörenberg, H.J. Mang, Nucl. Phys. A 136, 465 (1969) 